\section{Welchen gesellschaftlichen Beitrag kann Monitoring bei der Endlagerung radioaktiver Abfälle leisten?}

\author{
Eine „systemische“ Auseinandersetzung \\ mit dem TATuP-Schwerpunkt „Moni- \\ toring als soziale Innovation bei der \\ Endlagerung radioaktiver Abfälle"1
}

von Diana Gallego Carrera, ZIRIUS, Universität Stuttgart

\begin{abstract}
Die Endlagerung radioaktiver Abfälle ist eine höchst konfliktbehaftete Aufgabe. Im Umgang mit diesem Konflikt eine Strategie zu finden, die zum einen dem Stand von Wissenschaft und Technik entspricht und zum anderen ethisch-moralisch vertretbar ist, stellt eine große Herausforderung dar. In den Schwerpunktbeiträgen dieser Zeitschrift (3/2012) wurde das „soziale Monitoring“ als mögliche Lösungsoption diskutiert. Basierend auf den Annahmen dieses Themenschwerpunktes, widmet sich dieser Artikel der Frage, welchen Beitrag das soziale Monitoring zur Endlagerung radioaktiver Abfälle tastsächlich leisten kann. Die Beantwortung erfolgt aus systemischer Perspektive, die verdeutlicht, dass systemische Risiken ein wesentliches Kennzeichen der Moderne sind. Der Umgang mit innen erfordert ein vorsorgeorientiertes und umfassendes Risikomanagement.
\end{abstract}

\section{Einleitung}

Der TATuP-Schwerpunkt „Monitoring als soziale Innovation bei der Endlagerung radioaktiver Abfälle" beleuchtet und diskutiert den Themenkomplex mittels interdisziplinärer Expertise und im Hinblick auf die Annahme, dass ,... jedes Monitoring mehr oder minder komplexe gesellschaftliche Prozesse beinhaltet" (Hocke et al. 2013, S. 6). Die Autoren nehmen sich hierbei eines kaum überschaubaren Sachverhaltes an. Denn nicht ohne Grund wird die Endlagerung radioaktiver Abfälle von einem der Autoren als ,wicked problem“, also als ein verworrenes, vielschichtiges Problem bezeichnet (Brunnengräber et al. 2013, S. 59ff.).
Die Frage, welchen Beitrag ein gesellschaftliches Monitoring zur Lösung dieses ,wicked problems“ leisten kann, soll in diesem Artikel aus systemischer Perspektive erörtert werden. Ausgangspunkt der Erörterung ist die Debatte um die Endlagerung radioaktiver Abfälle in Deutschland. Hierbei gilt es, in einem ersten Schritt zu klären, warum dieses Thema ein ,wicked problem“ ist, um dann in einem zweiten Schritt zu prüfen, ob ein ,gesellschaftliches Monitoring" ein geeignetes Instrument im Umgang mit dieser Problematik sein kann.

\section{Bestimmung der Debatte um die Endlagerung radioaktiver Abfälle}

Der Standortfindungsprozess für die Endlagerung radioaktiver Abfälle in Deutschland ist seit jeher mit Konflikten behaftet. Ein wesentlicher Grund hierfür kann in der Vielzahl beteiligter Akteure gesehen werden, die über konträre Interessen, Handlungsoptionen und Zielvorstellungen bei der Lösungsfindung verfügen (Schetula/ Gallego Carrera 2012). Ergänzend zu diesem Konglomerat an Akteuren wird die Entsorgungsdebatte durch die Thematik selbst angefacht (Zilleßen 1998, S. 12f.): Sie spricht die Beziehung zwischen Mensch und Umwelt an und ist durch eine hohe Komplexität, Unabwägbarkeiten und Ungewissheit bestimmt. Die Endlagerung geht inhaltlich und zeitlich über politische und administrative Zuständigkeiten hinaus, potenzielle Folgen der Endlagerung überschreiten zeitliche und räumliche Grenzen. Ferner berührt die Thematik das Gemeinwohl, zeigt somit also einen Einfluss auf ganze Gesellschaften.

Anhand dieser Merkmale ist unschwer zu erkennen, wie vielschichtig (,,wicked“) das Thema der Endlagerung radioaktiver Abfälle ist. Diese Vielschichtigkeit ist es auch, die eine hohe Unsicherheit im Umgang mit dieser Thematik aufkommen lässt. Die Unsicherheit in der Endlagerdebatte bezieht sich hierbei nicht nur auf die Art und Weise der Endlagerung radioaktiver Abfälle, sondern auch auf die Bewertung von Risiken (Gallego Carrera/Schenkel 2009 bzw. Gallego Carrera/Hampel 2013). Bedenkt man, dass ein Risiko laut Luhmann ,...die Folge des eigenen Handelns... (ist) ${ }^{26 ،}$ (ebd. 1988, S. 269) so wird klar, warum ein formell (groß-)techni- 
scher Vorgang, wie die Endlagerung radioaktiver Abfälle, nicht ohne die Beachtung von gesellschaftlichen Prozessen vonstattengehen kann: Denn festzuhalten gilt, dass hinter jedem Risiko menschliche Entscheidungsprozesse stehen.

\section{Technische Risiken als Produkt menschlicher Entscheidungen}

Laut Beck (1986) sind wissenschaftlich-technisch erzeugte Risiken untrennbar mit modernen Gesellschaften, den sog. „Risikogesellschaften“ verbunden. Wissenschaftlich-technische Risiken werden nach dieser These zum konstituierenden Merkmal der Gesellschaften, indem sie deren Strukturen, Verhältnisse und Entwicklungen beeinflussen. Damit wissenschaftlich-technische Risiken ein derartiges gesellschaftliches Einflusspotenzial erreichen können, bedarf es organisierter Prozesse, die technologische Entwicklungen als Zusammenspiel der gesellschaftlichen Syste$\mathrm{me}^{3}$ forcieren. Dieses „Zusammenspiel“ lässt sich tagtäglich an den Orten der Wissensproduktion erkennen. Dort erfolgt die Wissensgenerierung beispielsweise wie folgt: Das politische System gibt einen Auftrag an das Wissenschaftssystem. Dieses bedarf der Technik und des praktischen Austausches seitens des Wirtschaftssystems, und das Wirtschaftssystem wird seinerseits vom politischen System gefördert. Diese Verwebung der einzelnen Systeme, wie Politik, Wissenschaft und Wirtschaft, ist einerseits notwendig für gesellschaftliche Innovationen, denn sie ermöglicht es, dass in einem beschleunigten Verfahren technische Errungenschaften erzeugt und neues Wissen generiert werden kann. Andererseits ist jedoch auch zu erkennen, dass sich diese technischen Errungenschaften primär durch Unabgeschlossenheit und Verbesserungsfähigkeit auszeichnen. ${ }^{4}$

Hinzu kommt, dass ein notwendig hoher Vernetzungsgrad vielfach nicht-intendierte Nebenfolgen (in anderen Systemen) nach sich zieht. Da der Vernetzungsgrad aber auch bedeutend für die Beherrschbarkeit von Technologien ist (Perrow 1987, S. 95ff.), kann laut Bechmann eine Zunahme katastrophaler Schäden als unmittelbare Folge der beschleunigten Technisierung der Gesellschaft postuliert werden (Bechmann 1990, S. 123).

\section{Das Systemische am Risiko}

Diese, im vorherigen Abschnitt aufgeführte Verzahnung von wissenschaftlich-technisch erzeugten Risiken mit kaum abschätzbaren Nebenfolgen, ist ein wesentliches Merkmal der stark vernetzten modernen Gesellschaften und ihrer ineinander verwobenen Systeme. Solche systemübergreifenden Risiken werden daher auch ,systemische Risiken" genannt. ${ }^{5}$

Systemische Risiken weisen in der Regel die folgenden Eigenschaften auf (Renn et al. 2007, S. 176f.):

- Sie strahlen auf andere, (in-)direkt vernetzte Systeme aus. Deren Funktionen oder Leistungen werden entweder gestört oder setzen aus (Ausstrahlungsaspekt).

- Sie verfügen über ein hohes Maß an Komplexität der Ursache-Wirkungs-Ketten, die oft nicht in ihren Verästelungen bekannt und beherrschbar sind (Komplexitätsaspekt).

- Im Umgang mit systemischen Risiken herrscht ein hohes Maß an Unsicherheit vor, das sich vielfach nicht hinreichend qualifizieren lässt (Unsicherheitsaspekt).

- Sie weisen ein hohes Maß an Mehrdeutigkeit hinsichtlich der zu erwartenden Konsequenzen und ihrer Bewertung auf (Ambiguitätsaspekt).

\section{Die Endlagerung radioaktiver Abfälle als systemisches Risiko}

Die zuvor aufgeführten Erkenntnisse um das $\mathrm{Zu}$ sammenspiel von gesellschaftlichen Systemen und wissenschaftlich-technischen Errungenschaften sind wesentlich, will man die Debatte um die Endlagerung radioaktiver Abfälle als einen gesellschaftlichen Prozess verstehen. Denn die Endlagerung vereint alle gesellschaftlich relevanten Merkmale systemischer Risiken in sich:

- Ausstrahlungsaspekt: Die Endlagerung radioaktiver Abfälle berührt Fragen der ökologischen, ökonomischen, juristischen, technischen, politischen und sozialen Systeme.

- Unsicherheitsaspekt: Die Tatsache, dass die zuvor aufgeführten Systeme wechselseitig agieren, impliziert, dass sekundäre und tertiäre nicht-intendierte Nebenfolgen verstärkt 
auftreten können. Bezüglich deren Eintrittswahrscheinlichkeit und Ausmaß herrscht hohe Unsicherheit vor.

- Komplexitätsaspekt: Vorgänge bei der Endlagerung radioaktiver Abfälle wirken sich unmittelbar auf andere Bereiche aus. So betrifft beispielsweise das Ökotoxizitätspotenzial gesetzlich festgelegte Grenzwerte, technische Herausforderungen sowie potenzielle Eingriffe in das Ökosystem.

- Die Endlagerung radioaktiver Abfälle zeichnet sich durch eine hohe Ambiguität in ihren Konsequenzen aus. Diese stellt wiederum eine besondere Anforderung an das Risikomanagement dar. Denn das Schaffen von Institutionen, die Schäden regulieren und vermeiden, ist schwierig, wenn nicht abschätzbar ist, welche Schäden überhaupt und in welchem Ausmaß auftreten können.

Daher stellt sich also die Frage, wie es unter hoher Unsicherheit gelingen kann, vernünftige Lösungsoptionen $\mathrm{zu}$ entwickeln, die einerseits so gut wie möglich auf faktischer Basis abgesichert und andererseits gesellschaftlich-moralisch tragfähig sind. Für die Endlagerung radioaktiver Abfälle könnte ein gesellschaftliches Monitoring ein richtiger Schritt in diese Richtung sein.

\section{Monitoring im Lichte der Endlagerung radioaktiver Abfälle}

Der Begriff „Monitoring“ bezieht sich im Endlagerkontext primär auf die technische Erfassung von Daten zur Entwicklung eines Endlagers und dessen Umgebung (Hocke et al. 2013, S. 5; ähnlich auch Grunwald 2013, S. 3; Kallenbach-Herbert/ Alt 2013, S. 18). Dies impliziert die Wiederholung technischer und geologischer Messungen über lange Zeiträume hinweg. Das wiederholte Messen der Daten dient hierbei als Entscheidungsgrundlage für oder wider eine Option. Da Entscheidungen im Spannungsfeld von Technologie und Geologie jedoch auch immer Auswirkungen auf das soziale Umfeld haben, schlagen Hocke et al. (2013) eine Differenzierung des Monitoringbegriffs nach sozialem und technischem Monitoring vor. Im Gegensatz zum technischen Monitoring richtet sich das soziale Monitoring nicht auf die Erhebung von
Daten, sondern betrachtet die gesellschaftlichen Prozesse, die notwendig sind, um technische Daten in Handlungsoptionen umzusetzen (Hocke et al. 2013, S. 6). Dieser am „Vorsorgeprinzip“ (ebd. S. 5) orientierte Ansatz wird dem Anspruch gerecht, dass sich Bedingungen infolge der für die Endlagerung anvisierten langen Zeiträume ändern können oder aber Entwicklungen auftreten, die in ihrem Umfang und Ausmaß bis dato nicht abschätzbar sind.

Vor diesem Hintergrund erscheint es umso eindrücklicher, dass in Deutschland bislang keine öffentlichen Diskurse zum Thema „Monitoring“ und dessen potenzieller Notwendigkeit stattgefunden haben (MoDeRn 2011). Bislang gibt es somit auch keine detaillierten und gültigen Empfehlungen dahingehend, wie ein Monitoring, das explizit gesellschaftspolitische Anforderungen mitberücksichtigt, durchzuführen ist (Kallenbach-Herbert/Alt 2013, S. 15). Dabei scheinen die Vorteile eines solchen Monitorings auf der Hand zu liegen, denn es ermöglicht in flexibler Art und Weise, Sachverhalte und Zusammenhänge zu erkennen, zu verstehen, zu bewerten und entsprechend zu handeln. Die Autoren des Themenschwerpunktes „Monitoring als soziale Innovation bei der Endlagerung radioaktiver Abfälle" sehen noch weitere Vorteile, die hier nur kurz angerissen werden können: Sie bewerten beispielsweise die Kontrolle über sicherheitsrelevante Aspekte (Appel/Kreusch 2013, S. 52), vertrauensbildende Maßnahmen und Transparenz (Wimmer et al. 2013, S. 30) oder die Integration moralischer Aspekte in die technische Arbeit (Bergmanns et al. 2013, S. 26) positiv.

Voraussetzung für solch positive Wirkung eines Monitorings ist jedoch, dass notwendige Entscheidungsgrundlagen vorhanden sind und dass Einigkeit zur Dateninterpretation und Setzung der Maßstäbe, an denen die Daten gemessen werden, vorherrscht. Doch genau hierin liegt das Problem. Denn welche Kennwerte sollen in ein Monitoring einfließen und wie können diese zuverlässig interpretiert werden? Insbesondere im Bereich der Endlagerung radioaktiver Abfälle stellt sich die Frage nach räumlich und zeitlich hinreichender Aussagekraft der Daten zum Systemverhalten (Appe1/Kreusch 2013, S. 57). Auch ist gegenwärtig ein Monitoring nur für begrenzte Phasen der 
Endlagerung denkbar, etwa bei der Erkundungs-, Errichtungs- und Betriebsphase. Für ein mögliches Monitoring nach dem Verschluss der Anlage sind hingegen die technischen und zeitlichen Bedingungen unklar (Wimmer et al. 2013, S. 31).

Hieraus lässt sich ableiten, dass für ein erfolgreiches Monitoring die Zielsetzung und Randbedingungen zur Datenerhebung und Interpretation frühzeitig festgelegt werden müssen. Womöglich bedarf es eines etappenweisen Vorgehens, um neu aufkeimende Unsicherheiten durch zu lange Zeiträume in der Zielsetzung einzudämmen. Hierbei ist eine frühe Einbeziehung der Bevölkerung vonnöten, so wie sie beispielsweise ansatzweise bereits beim Monitoring der Schachtanlage Asse II durchgeführt wird (Regenauer/Wittwer 2013, S. 33ff.). Hier wird die technische Datenanalyse von umfassenden Öffentlichkeitsinformationskampagnen begleitet. Doch eine Öffentlichkeitsinformationskampagne allein kann nicht das Ziel eines sozialen Monitorings sein. Der Konflikt um die Endlagerung radioaktiver Abfälle verlangt vielmehr nach einem strukturell fairen und diskursiven Verfahren, das die Werte der beteiligten Akteure berücksichtigt und Interessen auf faktischer Basis diskutiert. Komplexe Sachverhalte sollten hierbei der Öffentlichkeit in Form von Ausgestaltungsmöglichkeiten und Mitspracherecht zugänglich gemacht werden. Dieser sog. ,participatory turn“ (Sundqvist/Elam 2010) bedarf jedoch der Schaffung von entsprechenden Institutionen, die mittels eines übergeordneten Risikomanagements partizipative Prozesse planen und umsetzen können.

Dieses übergeordnete Risikomanagement ist notwendig, da die Endlagerung radioaktiver Abfälle systemische Risiken in sich birgt, die mit systemimmanenten Managementinstitutionen nicht gefasst werden können. Vielmehr gilt es nun, Koordination, Kooperation und Kommunikation zwischen den verschiedenen Gesellschaftsbereichen zu stärken (Renn/Keil 2008), um Risiken in ihrer Wechselwirkung zwischen physischen Konsequenzen, finanziellen Absicherungen und ihren psychologischen, sozialen und kulturellen Auswirkungen zu betrachten (Zinn/ Taylor-Gooby 2006). Aus der Betrachtung von Risiken leiten sich schließlich Handlungsoptionen $a b$. Deren Abwägung sollte hierbei den Stand des
Wissens ebenso berücksichtigen wie ethische und moralische Fragestellungen. Solch eine Aufgabe lässt sich nur als „Gemeinschaftsprojekt" der einzelnen Systeme bewältigen. Die Verknüpfung von technischem und sozialem Monitoring bietet die Option für ein derartiges systemübergreifendes Management. Denn die Kopplung beider Optionen ermöglicht es, die technisch gewonnenen Informationen in ein kooperatives, institutionalisiertes Kontroll- und Entscheidungsverfahren einzubinden (ähnliche Argumentation Kuppler/ Hocke 2013, S. 43). Ein zu institutionalisierendes, integrierendes Risikomanagement vermag durch das Monitoring die Kopplung verschiedener Wissensbestände zu gewährleisten und kann durch eine diskursive Verschränkung der Handlungsbereiche dem mehrdeutigen Charakter systemischer Risiken mehr Kontur verleihen (Renn/Keil 2008). Die Umsetzung neuer Erkenntnisse kann dann flexibel und systemübergreifend erfolgen.

\section{Schlussbetrachtung}

Dieser Text setzte sich mit dem Themenschwerpunkt „Monitoring als soziale Innovation bei der Endlagerung radioaktiver Abfälle" auseinander. Ausgangspunkt war hierbei, die dem Themenschwerpunkt entnommene Bezeichnung ,wicked problems" (Brunnengräber et al. 2013, S. 59) für die Endlagerung radioaktiver Abfälle. Diese Bezeichnung erscheint passend, da sich die Endlagerung radioaktiver Abfälle als mehrdimensionaler und vielschichtiger Konflikt darstellt. Grundlegend hierfür sind systemische Risiken, die sich primär durch vier Aspekte auszeichnen: Unsicherheit in der Datenerfassung und -abschätzung, Komplexität des Sachverhaltes, Ausstrahlung in andere Systeme mit sekundären und tertiären Folgewirkungen und Ambiguität der auftretenden Probleme. Dieses Konglomerat verlangt nach Lösungsoptionen, die einerseits so gut wie möglich auf faktischer Basis abgesichert werden und andererseits gesellschaftlich-moralisch tragfähig sind.

Für die Endlagerung radioaktiver Abfälle könnte ein soziales Monitoring in Ergänzung zum technischen Monitoring ein Schritt in die richtige Richtung sein. Während das technische Monitoring die Erhebung von Daten forciert, betrachtet das soziale Monitoring die gesellschaft- 
lichen Prozesse, die notwendig sind, um technische Daten in sozial tragfähige Handlungsoptionen umzusetzen. Durch die Verquickung von faktischer Datenbasis und gesellschaftlichen Umsetzungsoptionen kann es gelingen, den ambiguosen Charakter der systemischen Risiken einzugrenzen. Hierzu bedarf es jedoch eines ebenbürtigen Gleichschrittes der Integration divergierender Wissensbestände und diskursiven, systemübergreifenden Handlungsbereichen.

Diese Verknüpfung von Diskurselementen und faktischen Wissensbeständen vermag durch ein institutionalisiertes Risikomanagement gelingen, welches die Umsetzung neuer Erkenntnisse systemübergreifend vorantreibt. Dieses ,systemübergreifende Element" ist eine große Herausforderung für die Gesellschaft, da herkömmliche Institutionen zur Abfederung von systemischen Risiken (wie z. B. Versicherungen) nicht mehr greifen. Innovationen, die vorsorgeorientiert einen Beitrag zur partizipativen Technikgestaltung leisten, sind daher gefragt. Die Kopplung von technischem und sozialem Monitoring denkt diese Innovationen bereits an. Ihre Umsetzung scheint gegenwärtig jedoch erst in der Startphase zu stecken. Eine weitere Auseinandersetzung mit diesem Thema lohnt sich, denn systemische Risiken bzw. ,wicked problems" werden auch in Zukunft ein wesentliches Merkmal moderner Gesellschaften sein.

\section{Anmerkungen}

1) In: „Technikfolgenabschätzung - Theorie und Praxis“" (TATuP) 3/21 (2012), S. 5 -72

2) Das Risiko steht im Gegensatz zur Gefahr, die ein Ereignis bezeichnet, welches unabhängig vom eigenen Willen eintritt oder nicht (Luhmann 1991, S. 30f.).

3) Systeme entstehen aus der Wechselwirkung aufeinander bezogenen Handelns von Individuen, Gruppen oder Organisationen (Hillmann 1994, S. 857).

4) Produktzyklen werden kürzer. Denn kaum scheint ein technisches Produkt am Markt etabliert zu sein, so wird schon eine neuere, effizientere und schnellere Variante erprobt.

5) Das „systemische Risiko“ ist ein Terminus, der ursprünglich der Finanzwelt entstammte und dort das Finanzsystem an sich oder die Ökonomie als Ganzes durch entsprechende Domino-Effekte beeinflusste (Renn/Keil 2008).

\section{Literatur}

Appel, D.; Kreusch, J., 2013: Sicherheitstechnische und gesellschaftliche Aspekte von Monitoring bei der Endlagerung radioaktiver Abfälle mit Option ihrer Rückholbarkeit. In: Technikfolgenabschätzung - Theorie und Praxis 21/3 (2012), S. 52-58

Bechmann, G., 1990: Großtechnische Systeme. Risiko und gesellschaftliche Unsicherheit. In: Halfman, J.; Japp, K.-P. (Hg.): Riskante Entscheidungen und Katstrophenpotenziale. Elemente einer soziologischen Risikoforschung. Opladen, S. 123-149

Beck, U., 1986: Risikogesellschaft. Auf dem Weg in eine andere Moderne. Frankfurt a. M.

Bergmanns, A.; Elam, M.; Simmons, P. et al., 2013: Perspectives on Radioactive Waste Repository Monitoring. Conformation, Compliance, Confidence Building, and Societal Vigilance. In: Technikfolgenabschätzung - Theorie und Praxis 21/3 (2012), S. 22-27

Brunnengräber, A.; Mez, L.; Di Nucci, M.R. et al., 2013: Nukleare Entsorgung: Ein ,wicked“ und höchst konfliktbehaftetes Gesellschaftsproblem. In: Technikfolgenabschätzung - Theorie und Praxis 21/3 (2012), S. 59-65

Gallego Carrera, D.; Schenkel, W., 2009: Sachplan Geologische Tiefenlager. Kommunikation mit der Gesellschaft. Wissenschaftlicher Schlussbericht für das Bundesamt für Energie. Zürich

Gallego Carrerra, D.; Hampel, J., 2013: Die Situation der Kernenergie nach Fukushima - Wahrnehmung der Öffentlichkeit und politische Entscheide. In: Internationale Zeitschrift für Kernenergie 58/3 (2013), S. $175-180$

Grunwald, A., 2013: Editorial. In: Technikfolgenabschätzung - Theorie und Praxis 21/3 (2012), S. 3

Hillmann, K.-H., 1994: Wörterbuch der Soziologie. Stuttgart

Hocke, P.; Bergmans, A.; Kuppler, S., 2013: Einführung in den Schwerpunkt. In: Technikfolgenabschätzung - Theorie und Praxis 21/3 (2012), S. 5-9

Kallenbach-Herbert, B.; Alt, St., 2013: Monitoring als Baustein für die Entscheidungsfindung in Endlagerprojekten. In: Technikfolgenabschätzung - Theorie und Praxis 21/3 (2012), S.15-21

Kuppler, S.; Hocke, P., 2013: Monitoring in einem Pilotlager. Kontrollierte Deponierung von Nuklearabfällen im Konzept eines Schweizer Tiefenlagers. In: Technikfolgenabschätzung - Theorie und Praxis 21/3 (2012), S. 43-51

Luhmann, N., 1988: Die Wirtschaft der Gesellschaft. Frankfurt a. M.

Luhmann, N., 1991: Soziologie des Risikos. Berlin 
MoDeRn-Monitoring Developments for Safe Repository Operation and Staged Closure, 2011: National Monitoring Contexts. Summary Report; http://www. modern-fp 7.eu/fileadmin/modern/docs/Reports/ MoDeRn_MonitoringContext_SummaryReport.pdf (download 11.4.13)

Nennen, H.-U.; Garbe, D., 1996: Das Expertendilemma: zur Rolle wissenschaftlicher Gutachter in der öffentlichen Meinungsbildung. Berlin

Perrow, Ch., 1987: Normale Katastrophen. Die unvermeidbaren Risiken der Großtechnik. Frankfurt a. M.

Regenauer, U.; Wittwer, Chr., 2013: Monitoring der Schachtanlage Asse II. In: Technikfolgenabschätzung - Theorie und Praxis 21/3 (2012), S. 33-42

Renn, O.; Keil, F., 2008: Systemische Risiken: Versuch einer Charakterisierung. In: GAIA 17/4 (2008), S. 349-354

Renn, O.; Schweizer, P.-J.; Dreyer, M. et al., 2007: Risiko. Über den gesellschaftlichen Umgang mit Unsicherheit. München

Schetula, V.; Gallego Carrera, D., 2012: Möglichkeiten und Grenzen der Bürgerbeteiligung: Das Fallbeispiel der Endlagerung radioaktiver Abfälle. In: Müller, M. (Hg.): Endlagersuche: Auf ein Neues? Der Weg zu einem gerechten und durchführbaren Verfahren. Loccumer Protokoll 25/12. Rehburg-Loccum, S. 125-136

Sundqvist, G.; Elam, M., 2010: Public Involvement Designed to Circumvent Public Concern? The "Participatory Turn" in European Nuclear Activities. In: Risk, Hazards \& Crisis in Public Policy 1/4

Wimmer, H.; Brammer, K.-J.; Köbl, M., 2013: Monitoring im Endlager. Notwendig für die Akzeptanz? Anmerkungen aus Sicht eines Betreibers für Zwischenlager. In: Technikfolgenabschätzung - Theorie und Praxis 21/3 (2012), S. 28-32

Zilleßen, H., 1998: Mediation: Kooperatives Konfliktmanagement in der Umweltpolitik. Opladen

Zinn, J.O.; Taylor-Gooby, P., 2006: Risk as an Interdisziplinary Research Area. In: Zinn, J.O.; Taylor-Gooby, P. (Hg.): Risk in Social Science. Oxford, UK, S. $20-53$

\section{Kontakt}

Diana Gallego Carrera

ZIRIUS - Zentrum für Interdisziplinäre Risiko- und Innovationsforschung der Universität Stuttgart Seidenstraße 36, 70174 Stuttgart

E-Mail: diana.gallego@sowi.uni-stuttgart.de

\section{Autorenhinweise}

Wir bitten alle Autorinnen und Autoren, die ein Manuskript bei TATuP einreichen, die folgenden Hinweise zu beachten:

Umfang: Eine Druckseite umfasst max. 3.500 Zeichen (ohne Leerzeichen). Für den Umfang eines Beitrags ist die Rubrik, in der er erscheint, ausschlaggebend. Genauere Angaben erhalten die Autoren von der Redaktion.

Abstract: Autoren, deren Beiträge im Themenschwerpunkt des Heftes oder in den Rubriken TA-Konzepte und -Methoden und Diskussionsforum sowie TA-Projekte erscheinen, werden gebeten, ihrem Beitrag ein Abstract voranzustellen, in dem eine kurze inhaltliche Übersicht über den Beitrag gegeben wird. Die Länge dieses Abstracts sollte 780 Zeichen (ohne Leerzeichen) nicht überschreiten.

Abbildungen, Diagramme und Tabellen: Abbildungen und Tabellen sind sowohl in das eingereichte Manuskript einzufügen sowie auch getrennt von der ersten Fassung des Manuskripts einzusenden. Abbildungen und Tabellen bitte mit Überschrift und Quellenangabe versehen. Wurden sie vom Autor selbst erstellt, bitte die Formulierung „eigene Darstellung“ als Quellenangabe verwenden Zum Format: Tabellen sind als Word-Datei, Diagramme in Excel und Abbildungen in Adobe Illustrator oder Powerpoint zu liefern. Sollten Sie lediglich andere Formate zur Verfügung haben, wenden Sie sich bitte frühzeitig an die Redaktion. Aus Gründen der Seitenplanung und des Layouts liegt die Entscheidung über die endgültige Größe und Platzierung der Abbildungen und Tabellen innerhalb des Beitrags bei der Redaktion.

Bibliografische Angaben: Die zitierte Literatur wird am Ende des Beitrags als Liste in alphabetischer Reihenfolge angegeben. Im Text selbst geschieht dies in runden Klammern (z. B. Wiegerling 2011); bei Zitaten ist die Seitenangabe hinzuzufügen (z. B. Fink/Weyer 2011, S. 91). Bei den Angaben in der Literaturliste orientieren Sie sich bitte an folgenden Beispielen:

Monografien: Wiegerling, K., 2011: Philosophie intelligenter Welten. München

Bei Aufsätzen: Fink, R.D.; Weyer, J., 2011: Autonome Technik als Herausforderung der soziologischen Handlungstheorie. In: Zeitschrift für Soziologie 40/2 (2011), S. 91-111

Bei Beiträgen in Sammelbänden: Mehler, A., 2010: Artifizielle Interaktivität. Eine semiotische Betrachtung. In: Sutter, T.; Mehler, A. (Hg.): Medienwandel als Wandel von Interaktionsformen. Heidelberg

Bei Internet-Quellen: Waterfield, J., 2006: From Corporation to Transnational Pluralism. London; http:// www.plugin-tot.com (download 12.3.09) 